\title{
Dos regímenes de dosis de nifedipino para el manejo del trabajo de parto prematuro: un estudio randomizado controlado (1)
}

\author{
Nassar AH, Abu-Musa AA, Awwad J, Khalil A, Tabbara J, Usta IM. Two dose regimens \\ of nifedipine for management of preterm labor: a randomized controlled trial. Am J Peri- \\ natol 2009;26(8):575-81.
}

Análisis crítico: Jorge Carvajal C., PhD, Claudio Vera PG., MSc.

Unidad de Medicina Materno Fetal, División de Obstetricia y Ginecología, Pontificia Universidad Católica de Chile.

\section{RESUMEN (1)}

Comparamos dos regímenes de dosis del tocolítico oral nifedipino. Mujeres con embarazo único admitidas en trabajo de parto prematuro (24 a 34 semanas) fueron randomizadas a dosis alta (DA) de nifedipino $(n=49 ; 20 \mathrm{mg}$ dosis de carga repetidos en 30 minutos, dosis diaria de 120 a $160 \mathrm{mg}$ de nifedipino de liberación lenta por 48 horas, seguido de 80 a $120 \mathrm{mg}$ diarios hasta las 36 semanas) o dosis baja (DB) de nifedipino ( $n=53 ; 10 \mathrm{mg}$ hasta 4 dosis cada 15 minutos, diariamente 60 a $80 \mathrm{mg}$ de nifedipino de liberación prolongada por 48 horas, seguido de $60 \mathrm{mg}$ diarios hasta las 36 semanas). Quiescencia uterina a las 48 horas (resultado primario), parto a las 48 horas, 34 y 37 semanas, y trabajo de parto prematuro recurrente fueron similares. La edad gestacional al parto fue mayor en DA $(36,0 \pm 2,8$ versus $34,7 \pm 3,7$ semanas, $p=0,049$ ). Tratamiento de rescate fue requerido más veces en $D B(24,5$ versus $50,9 \%$, OR=0,3; 95\% IC 0,1-0,7). Efectos adversos maternos, peso de nacimiento, admisión a unidad de cuidados intensivos neonatales y morbilidad neonatal compuesta fueron similares. Sin embargo, necesidad de ventilación mecánica y estadía en neonatología fue más corta en DA. DA de nifedipino no parece tener ventadas sobre DB en alcanzar quiescencia uterina a las 48 horas. Estudios adicionales deberán encontrar la dosis y formulación óptima de nifedipino como tocolítico.

\section{ANALISIS DE LA INVESTIGACIÓN}

\section{A. Relevancia clínica de la investigación}

En los últimos años el nifedipino ha tomado el primer lugar como la droga de elección para el manejo del trabajo de parto prematuro, sin embargo se desconoce cuál es el régimen de tratamiento óptimo con nifedipino como tocolítico. La incidencia de parto prematuro fluctúa entre un 6 a $10 \%$. Un tercio de estos prematuros corresponden al grupo parto prematuro idiopático, es decir aquellos originados en un inicio espontáneo del trabajo de parto prematuro (2). El tratamiento de pacientes en trabajo de parto prematuro incluye la administración de corticoides para inducir maduración pulmonar fetal y tocolíticos para reducir las contracciones uterinas. Un tocolítico ideal es aquel que no tiene efectos adversos para la madre y el feto, y permite una prolongación significativa del embarazo (reducción del parto prematuro o al menos reducción de la morbimortalidad perinatal asociada a prematurez). Lamentablemente los tocolíticos hoy disponibles parecen alejarse de este ideal. El único fármaco tocolítico que ha demostrado su capacidad de reducir la morbilidad perinatal asociada a prematurez es el nifedipino, sin embargo los artículos que prueban su utilidad utilizan dosis variadas del fármaco, incluyendo diferentes regímenes de tratamiento agudo y de mantención (3). 


\section{B. El estudio (1)}

Diseño: Investigación clínica controlada aleatorizada, abierta (sin ciego). Pacientes: Mujeres con embarazos únicos, membranas íntegras, en trabajo de parto activo, cursando entre 24 a 34 semanas de embarazo. Criterios de exclusión: dilatación mayor a $4 \mathrm{~cm}$, restricción de crecimiento fetal, malformaciones congénitas, estado fetal no tranquilizador, corioamnionitis, uso previo de tocolíticos en este embarazo, presión arterial menor a 90/50, enfermedades maternas (insuficiencia renal, disfunción hepática o enfermedad cardiaca y complicaciones obstétricas (PPO, DPPNI, SHE). Intervención: Dosis Alta: $20 \mathrm{mg}$ de nifedipino sublingual repetido en 30 minutos (si persisten CU). Dosis de mantención de 120-160 mg diarios usando nifedipino de liberación prolongada (30-40 mg/6 horas) por 48 horas. Luego, mantención con 80-120 mg/día hasta las 36 semanas. Comparación: Dosis Baja: $10 \mathrm{mg}$ de nifedipino sublingual, repetidos cada 15 minutos durante la primera hora (según necesidad). Dosis de mantención $60-80 \mathrm{mg} /$ día por 48 horas $(20 \mathrm{mg}$ cada 6-8 horas). Luego, mantención con 60 mg/día hasta las 36 semanas. Resultado primario evaluado: lograr quiescencia uterina dentro de 48 horas (definido como menos de 4 contracciones por horas sin cambios cervicales). Resultados secundarios evaluados: quiescencia uterina a las 6 horas, tiempo para alcanzar la quiescencia uterina, proporción de mujeres que no han tenido el parto dentro de 48 horas, 34 y 37 semanas, período de latencia, edad gestacional al parto y efectos adversos maternos. Variables neonatales: admisión a UCI neonatal, ventilación mecánica, síndrome de dificultad respiratoria (SDR), hemorragia intraventricular (HIV), enterocolitis necrotizante (ECN), ictericia neonatal, sepsis, estadía en $\mathrm{UCl}$, muerte neonatal. Morbilidad materna severa compuesta (dolor torácico, edema pulmonar, disnea e hipotensión). Morbilidad neonatal severa compuesta (HIV, SDR, ECN o sep- sis). Necesidad de tratamiento de rescate, definido como la necesidad de un tocolítico adicional (en el caso de dosis alta) o la necesidad de dosis alta en el grupo de dosis baja. Resultados: No hubo diferencias significativas en el resultado primario (Tabla I); si hubo diferencias significativas en algunos resultados secundarios que se muestran en la tabla.

\section{Análisis crítico}

Validez interna: El estudio aporta conocimiento nuevo, y relevante pues el régimen de tratamiento óptimo para la tocolisis con nifedipino es desconocido. El diseño del estudio es apropiado porque permite responder la pregunta planteada. Se explica claramente el cálculo de tamaño muestral, sin embargo este no fue cumplido (se reclutó al 50\% de las pacientes requeridas). La randomización fue apropiada. El análisis no se efectuó por intención de tratar, pues dos pacientes en el grupo dosis alta abandonaron el tratamiento y no fueron consideradas en el análisis. El seguimiento fue incompleto (el resultado de un neonato en cada grupo no fue conocido).

Comentario: Si bien el diseño es apropiado a la pregunta, el estudio posee fuentes importantes de sesgo: no se cumple el tamaño muestral y el análisis no fue según intención de tratar. La falta de un tamaño muestral apropiado aumenta el riesgo de error de tipo alfa (falso positivo) y de tipo beta (falso negativo), invalidando por completo los resultados del estudio. Dos pacientes del grupo dosis alta abandonan tratamiento y no son analizadas, sugiriendo que no toleraron la dosis alta de nifedipino. Estas pacientes debieron ser analizadas para respetar la randomización.

Conclusión: el tema del estudio es de gran importancia, apunta a un conocimiento indispensable para la práctica obstétrica, sin embargo esta investigación adolece de graves defectos en su implementación que incrementan notoriamente el riesgo

Tabla I

RESULTADOS DEL ESTUDIO

\begin{tabular}{lccc}
\hline Resultado primario & Dosis Alta & Dosis Baja & OR (IC 95\%) o valor p \\
Quiescencia uterina dentro de 48 horas & $71,4 \%$ & $56,6 \%$ & $1,9(0,9-4,3)$ \\
Resultados secundarios & & $58,5 \%$ & $2,8(1,2-6,6)$ \\
Quiescencia uterina dentro de 6 horas & $79,6 \%$ & $50,9 \%$ & $0,3(0,1-0,7)$ \\
Necesidad de tratamiento de rescate & $24,5 \%$ & $34,7 \pm 3,7$ & 0,049 \\
Edad gestacional al parto & $36,0 \pm 2,8$ & $10,3 \pm 13,7$ & 0,023 \\
Estadía en neonatología & $5,5 \pm 4,6$ &
\end{tabular}


de sesgo. Recomendamos no considerar estos resultados como válidos para nuestra práctica clínica.

\section{REFERENCIAS}

1. Nassar AH, Abu-Musa AA, Awwad J, Khalil A, Tabbara J, Usta IM. Two dose regimens of nifedipine for management of preterm labor: a randomized controlled trial. Am J Perinatol 2009;26(8):575-81.

2. Simhan HN, Caritis SN. Prevention of preterm delivery. N Engl J Med 2007;357(5):477-87.

3. King JF, Flenady VJ, Papatsonis DN, Dekker GA, Carbonne B. Calcium channel blockers for inhibiting preterm labour. Cochrane Database Syst Rev. 2003;(1):CD002255. 